\title{
Pion cloud and the sea of the nucleon
}

\author{
W. Melnitchouk \\ Jefferson Lab, 12000 Jefferson Avenue, Newport News, VA 23606, USA
}

\begin{abstract}
I review recent progress in understanding the structure of the nucleon sea and the role of the nucleon's pion cloud. In particular, I discuss the consequences of the pion cloud for the $\bar{d}-\bar{u}$ asymmetry in the proton, the neutron's electric form factor, and the proton's electric to magnetic form factor ratio.
\end{abstract}




\section{Introduction}

My interactions with Prof. Manoj Banerjee occurred mostly during my postdoc at the University of Maryland, between the years of 1995 and 1997. I was fortunate enough to have benefited from his vast knowledge of the quark structure of nucleons, particularly the constraints on hadronic structure from chiral symmetry [1].

One of my main interests at the time, as for much of my career, concerned the structure of the nucleon sea. I had spent a substantial part of my $\mathrm{Ph}$. D. work developing, with Prof. A. W. Thomas, the meson cloud model of deep inelastic scattering [2], in an attempt to identify the origin of the recently discovered $\bar{d}-\bar{u}$ asymmetry in the proton sea. Although not universally accepted at the time, the relevance of the meson (and in particular the pion) cloud in deep inelastic scattering (DIS) was later firmly established in the context of chiral perturbation theory [3-5], as a model-independent prediction of QCD.

In this article I review the role of chiral symmetry in observables which are sensitive to the chiral structure of QCD and are strongly affected by the pion cloud of the nucleon. In Sec. 2 I recall the derivation of the leading non-analytic behavior of the pion cloud contribution to the $\bar{d}-\bar{u}$ asymmetry in the proton, arising from the infra-red behavior of chiral loops. Predictions for the ratio of $\bar{d} / \bar{u}$ are made in Sec. 3 within a specific meson cloud model of the nucleon [2].

Within the same meson cloud framework one naturally expects a nonzero charge distribution in the neutron, and in Sec. 4 I discuss the role of the pion cloud in the neutron Sachs electric form factor [6]. With the recent intense interest in the $Q^{2}$ behavior of the proton's

electric to magnetic form factor ratio, I also briefly review in Sec. 5 suggestions about the possible role of chiral physics in this observable. Finally, in Sec. 6 I make some concluding remarks and outline future directions for the study of the nucleon sea.

\section{Chiral symmetry and the pion cloud}

Understanding the role of dynamical chiral symmetry breaking in hadron structure is one of the main challenges of strong interaction physics. On general grounds one can show that Goldstone boson loops make important contributions to hadronic properties such as 
masses, charge distributions and magnetic moments. In the chiral limit the charge radii of the proton and neutron, for example, are known to diverge as $\ln m_{\pi}$ [7]. Such non-analytic behavior as a function of quark mass [8] is a unique characteristic of Goldstone boson loops $[3]$.

While pions are known to play a crucial role in the structure and dynamics of the nucleon in low energy physics, there is no reason why the long-range tail of the nucleon should not also play a role at high energies. This was first postulated by Sullivan [9], who argued that DIS from the pion cloud of the nucleon contributes to the nucleon structure function at large $Q^{2}$.

As later pointed out by Thomas [10], if the proton's wave function contains an explicit $\pi^{+} n$ Fock state component, a DIS probe scattering from the virtual $\pi^{+}$, which contains a valence $\bar{d}$ quark, will automatically lead to an excess of $\bar{d}$ over $\bar{u}$ in the proton. A number of experiments subsequently verified that indeed $\bar{d}>\bar{u}[11-14]$, and many theoretical analyses followed attempting to describe the asymmetry in terms of the nucleon's pion cloud [15].

Until recently, however, no rigorous connection existed between the pion cloud models and the chiral properties of QCD, which prevented the fundamental importance of the pion contribution to the flavor asymmetry being universally accepted. This problem was addressed several years ago by Thomas et al. [3], who demonstrated that the nucleon's pion cloud gives rise to unique terms in the moments of $\bar{d}-\bar{u}$ that are non-analytic in the quark mass. The leading non-analytic (LNA) behavior of the excess number of $\bar{d}$ over $\bar{u}$ arises from the infra-red behavior of chiral loops in chiral effective theories, such as chiral perturbation theory [16], and is thus model independent.

Specifically, one finds for the LNA behavior of the lowest moment of $\bar{d}-\bar{u}[3]$ :

$$
\int_{0}^{1} d x(\bar{d}(x)-\bar{u}(x))_{\mathrm{LNA}}=\frac{2 g_{A}^{2}}{\left(4 \pi f_{\pi}\right)^{2}} m_{\pi}^{2} \log \left(m_{\pi}^{2} / \mu^{2}\right),
$$

where $g_{A}$ is the axial charge of the nucleon (understood to be taken in the chiral SU(2) limit, $m_{q} \rightarrow 0$ ), and $\mu$ is a mass parameter. This result also generalizes to higher moments, each of which has a non-analytic component, so that the $\bar{d}-\bar{u}$ distribution itself, as a function of $x$, has a model-independent, LNA component. The presence of non-analytic terms indicates that Goldstone bosons play a role which cannot be cancelled by any other physical process (except by chance and only at a particular value of $m_{\pi}$ ). Such insight is important when it 
comes to building models and developing physical understanding of a system.

\section{3. $\bar{d}-\bar{u}$ asymmetry in the proton sea}

Because gluons in QCD are flavor-blind, the perturbative process $g \rightarrow q \bar{q}$ gives rise to a sea component of the nucleon which is symmetric in the quark flavors. Although differences can arise due to different quark masses, because isospin symmetry is such a good symmetry in nature, one would expect that the sea of light quarks generated perturbatively would be almost identical, $\bar{u}(x) \approx \bar{d}(x)$.

It was therefore a major surprise to many when measurements by the New Muon Collaboration (NMC) at CERN [11] of the proton and deuteron structure functions suggested a significant excess of $\bar{d}$ over $\bar{u}$ in the proton. Indeed, it heralded a renewed interest in the role of nonperturbative QCD in DIS processes. While the NMC experiment measured the integral of the antiquark difference, subsequently the E866 experiment at Fermilab [13] mapped out the shape of the $\bar{d} / \bar{u}$ ratio over the $x$ range $0.02<x<0.345$.

The E866 Collaboration measured $\mu^{+} \mu^{-}$Drell-Yan pairs produced in $p p$ and $p d$ collisions, whose cross section in the parton model is proportional to:

$$
\sigma^{p h} \propto \sum_{q} e_{q}^{2}\left(q^{p}\left(x_{1}\right) \bar{q}^{h}\left(x_{2}\right)+\bar{q}^{p}\left(x_{1}\right) q^{h}\left(x_{2}\right)\right)
$$

where $h=p$ or $d$, and $x_{1}$ and $x_{2}$ are the light-cone momentum fractions carried by partons in the projectile and target hadron, respectively. Using isospin symmetry to relate quark distributions in the neutron to those in the proton, in the limit $x_{1} \gg x_{2}$ (in which $\bar{q}\left(x_{1}\right) \ll$ $\left.q\left(x_{1}\right)\right)$ the ratio of the deuteron to proton cross sections can be written:

$$
\frac{\sigma^{p d}}{2 \sigma^{p p}}=\frac{1}{2}\left(1+\frac{\bar{d}\left(x_{2}\right)}{\bar{u}\left(x_{2}\right)}\right) \frac{4+d\left(x_{1}\right) / u\left(x_{1}\right)}{4+d\left(x_{1}\right) / u\left(x_{1}\right) \cdot \bar{d}\left(x_{2}\right) / \bar{u}\left(x_{2}\right)} .
$$

The relatively large asymmetry found in these experiments, shown in Fig. 1, implies the presence of nontrivial dynamics in the proton sea which does not have a perturbative QCD origin.

The simplest and most obvious source of a nonperturbative asymmetry in the light quark sea is the chiral structure of QCD. One can consider a model in which the nucleon core consists of valence quarks, interacting via gluon exchange for example, with sea quark effects 


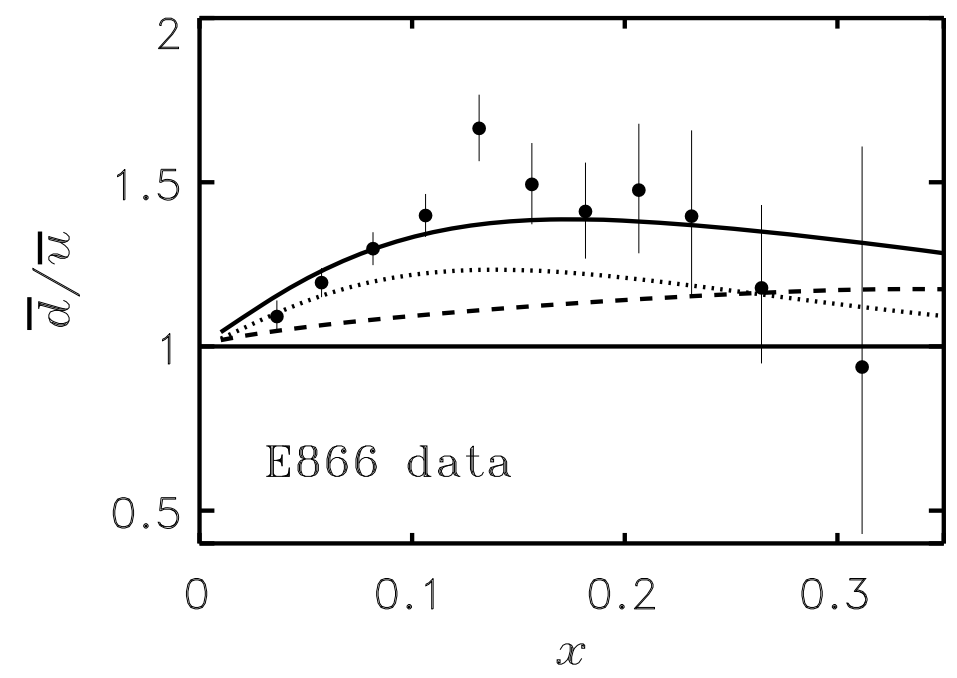

FIG. 1: Flavor asymmetry of the light antiquark sea, including pion cloud (dashed) and Pauli blocking effects (dotted), and the total (solid) [17].

introduced through the coupling of the core to $q \bar{q}$ states with pseudoscalar meson quantum numbers. Many variants of such a model exist — see Refs. [1, 2, 15, 18] for example.

In this framework the physical nucleon state (with momentum $P$ ) can be expanded (in the one-meson approximation) as a series involving bare nucleon and two-particle meson-baryon states $[2,15,18]$ :

$$
\begin{aligned}
& |N(P)\rangle_{\text {phys }}=\sqrt{Z}\left\{|N(P)\rangle_{\text {bare }}\right. \\
& \left.+\sum_{B, M} \int d y d^{2} \mathbf{k}_{\perp} g_{M N B} \phi_{B M}\left(y, \mathbf{k}_{\perp}\right)\left|B\left(y, \mathbf{k}_{\perp}\right) ; M\left(1-y,-\mathbf{k}_{\perp}\right)\right\rangle\right\}
\end{aligned}
$$

where $M=\pi, K, \cdots$ and $B=N, \Delta, \Lambda, \cdots$. The function $\phi_{B M}\left(y, \mathbf{k}_{\perp}\right)$ is the probability amplitude for the physical nucleon $N$ to be in a state consisting of a baryon $B$ and meson $M$, having transverse momenta $\mathbf{k}_{\perp}$ and $-\mathbf{k}_{\perp}$, and carrying longitudinal momentum fractions $y=k_{+} / P_{+}$and $1-y=\left(P_{+}-k_{+}\right) / P_{+}$, respectively. The bare nucleon probability is denoted by $Z$, and $g_{M N B}$ is the $M N B$ coupling constant. The one-meson approximation in Eq. (4) will be valid as long as the meson cloud is relatively soft $(Z \approx 1)$. It will progressively break down for harder $M N B$ vertex functions, at which point one will need to include two-meson and higher order Fock state components.

Because of the small pion mass, the $\pi N$ component of the Fock state expansion in Eq. (4) 
will be the most important (contributions from heavier mesons and baryons will be progressively suppressed with increasing mass). In the impulse approximation, DIS from the $\pi N$ component of the proton can then be understood in the IMF as the probability for a pion to be emitted by the proton, folded with the probability of finding the a parton in the pion [19]. For the antiquark asymmetry, this can be written as [15]:

$$
\bar{d}(x)-\bar{u}(x)=\frac{2}{3} \int_{x}^{1} \frac{d y}{y} f_{\pi N}(y) \bar{q}^{\pi}(x / y),
$$

where $\bar{q}^{\pi}$ is the (valence) quark distribution in the pion (e.g. $\bar{u}$ in $\pi^{+}$, normalized to unity), and the distribution of pions with a recoiling nucleon $(N \rightarrow \pi N$ splitting function) is given by $[9,10,20]$ :

$$
f_{\pi N}(y)=\frac{3 g_{\pi N N}^{2}}{16 \pi^{3}} \int \frac{d^{2} \mathbf{k}_{T}}{(1-y)} \frac{\mathcal{F}_{\pi N}^{2}\left(s_{\pi N}\right)}{y\left(M^{2}-s_{\pi N}\right)^{2}}\left(\frac{k_{T}^{2}+y^{2} M^{2}}{1-y}\right) .
$$

The invariant mass squared of the $\pi N$ system is given by:

$$
s_{\pi N}=\frac{k_{T}^{2}+m_{\pi}^{2}}{y}+\frac{k_{T}^{2}+M^{2}}{1-y},
$$

and for the functional form of the $\pi N N$ vertex function $\mathcal{F}_{\pi N}\left(s_{\pi N}\right)$ one can take a simple dipole parameterization, $\mathcal{F}_{\pi N}\left(s_{\pi N}\right)=\left(\left(\Lambda^{2}+M^{2}\right) /\left(\Lambda^{2}+s_{\pi N}\right)\right)^{2}$, normalized so that the coupling constant $g_{\pi N N}$ has its standard value $(=13.07)$ at the pole $\left(\mathcal{F}\left(M^{2}\right)=1\right)$. Note that the contribution from the pion cloud in Eq. (5) is a leading twist effect which scales with $Q^{2}$ - at leading order the $Q^{2}$ dependence of $\bar{d}-\bar{u}$ in Eq. (5) enters through the leading-twist quark distribution in the pion, $\bar{q}^{\pi}$.

One can easily generalize the above to include higher Fock state components [15, 20], most notably the $\Delta$. Because the dominant process there is $p \rightarrow \Delta^{++} \pi^{-}$, the $\Delta$ will actually cancel some of the $\bar{d}$ excess generated through the $\pi N$ component, although this will be somewhat smaller due to the larger mass of the $\Delta$.

The relative contributions are partly determined by the $\pi N N$ and $\pi N \Delta$ vertex form factor. The form factor cut-offs $\Lambda$ can be determined phenomenologically by comparing against various inclusive and semi-inclusive data, although the most direct way to fix these parameters is through a comparison of the axial form factors for the nucleon and for the $N-\Delta$ transition. Within the framework of the partially conserved axial current (PCAC) these form factors are directly related to the corresponding form factors for pion emission or absorption. The data on the axial form factor are best fit, in a dipole parameterization, 
by a $1.3(1.02) \mathrm{GeV}$ dipole for the axial $N(N-\Delta$ transition) form factor [15], which gives a pion probability in the proton of $\approx 13 \%(10 \%)$.

With these parameters Fig. 1 shows the $\bar{d} / \bar{u}$ ratio in the proton due to $\pi N$ and $\pi \Delta$ components of the nucleon wave function (dashed line) [17]. Data on the sum of the $\bar{u}$ and $\bar{d}$ (which is dominated by perturbative contributions) have been used to convert the calculated $\bar{d}-\bar{u}$ difference to the $\bar{d} / \bar{u}$ ratio. The results suggest that with pions alone one can account for about half of the observed asymmetry, leaving room for possible contributions from other mechanisms.

Another mechanism which could also contribute to the $\bar{d}-\bar{u}$ asymmetry is associated with the effects of antisymmetrization of $q \bar{q}$ pairs created inside the core [21]. As pointed out originally by Field and Feynman [22], because the valence quark flavors are unequally represented in the proton, the Pauli exclusion principle will affect the likelihood with which $q \bar{q}$ pairs can be created in different flavor channels. Since the proton contains 2 valence $u$ quarks compared with only one valence $d$ quark, $u \bar{u}$ pair creation will be suppressed relative to $d \bar{d}$ creation. In the ground state of the proton the suppression will be in the ratio $\bar{d}: \bar{u}=5: 4$.

Phenomenological analyses in terms of low energy models suggest that the contribution from Pauli blocking can contribute around half of the $\bar{d}$ excess observed empirically. Together with the integrated asymmetry from pions, the combined value for the integral of $\bar{d}-\bar{u}$ over $x$ of $\approx 0.12$ is in quite reasonable agreement with the experimental result, $0.100 \pm 0.018$ from E866 [13].

Although the combined pion cloud and Pauli blocking mechanisms are able to fit the E866 data reasonable well at small and intermediate $x(x<0.2)$, it is difficult to reproduce the apparent trend in the data at large $x$ towards zero asymmetry, and possibly even an excess of $\bar{u}$ for $x>0.3$. Unfortunately, the error bars are quite large beyond $x \sim 0.25$, and it will be important to have new Drell-Yan data at larger $x$ to clarify this [23].

A different approach to the problem makes use of semi-inclusive scattering, tagging charged pions produced off protons and neutrons. Taking the ratio of the isovector combination of cross sections for $\pi^{+}$and $\pi^{-}$production [24],

$$
\frac{N_{p}^{\pi^{+}+\pi^{-}}-N_{n}^{\pi^{+}+\pi^{-}}}{N_{p}^{\pi^{+}-\pi^{-}}-N_{n}^{\pi^{+}-\pi^{-}}}=\frac{3}{5}\left(\frac{u-d-\bar{d}+\bar{u}}{u-d+\bar{d}-\bar{u}}\right)\left(\frac{D+\bar{D}}{D-\bar{D}}\right),
$$

the difference $\bar{d}-\bar{u}$ can be directly measured provided the $u$ and $d$ quark distributions 
and fragmentation functions $D$ and $\bar{D}$ are known. A measurement of this ratio by the HERMES Collaboration [25] was found to be consistent with the Drell-Yan results, although the rapidly falling cross sections at large $x$ make semi-inclusive measurements beyond $x \sim 0.3$ challenging. On the other hand, the high luminosity and higher energy available at Jefferson Lab following the $12 \mathrm{GeV}$ Upgrade would allow the asymmetry to be measured well beyond $x \sim 0.3$ with relatively small errors.

\section{Neutron electric form factor}

If a pseudoscalar cloud of $q \bar{q}$ states plays an important role in the $\bar{d} / \bar{u}$ asymmetry, its effects should also be visible in other flavor-sensitive observables, such as electromagnetic form factors [26]. An excellent example is the electric form factor of the neutron, $G_{E}^{n}$, a nonzero value for which can arise from a pion cloud, $n \rightarrow p+\pi^{-}$. Although in practice other effects such as spin-dependent interactions due to one gluon exchange between quarks in the core [27] will certainly contribute at some level, it is important nevertheless to evaluate the consequences of the pion cloud model for all observables that may carry its signature.

To illustrate the sole effect of the pion cloud, all residual interactions between quarks in the core can be switched off, so that the form factors have only two contributions: one in which the photon couples to the virtual $\pi$ and one where the photon couples to the recoil nucleon:

$$
\mathcal{F}_{1,2}\left(Q^{2}\right)=\int_{0}^{1} d y\left(f_{1,2}^{(\pi)}\left(y, Q^{2}\right)+f_{1,2}^{(N)}\left(y, Q^{2}\right)\right) .
$$

The recoil nucleon contribution is described by the functions:

$$
\begin{aligned}
f_{1}^{(N)}\left(y, Q^{2}\right)= & \frac{3 g_{\pi N N}^{2}}{16 \pi^{3}} \int \frac{d^{2} \mathbf{k}_{\perp}}{y^{2}(1-y)} \frac{\mathcal{F}\left(s_{N \pi, i}\right) \mathcal{F}\left(s_{N \pi, f}\right)}{\left(s_{N \pi, i}-M^{2}\right)\left(s_{N \pi, f}-M^{2}\right)} \\
& \times\left(k_{\perp}^{2}+M^{2}(1-y)^{2}-(1-y)^{2} \frac{q_{\perp}^{2}}{4}\right), \\
f_{2}^{(N)}\left(y, Q^{2}\right)= & \frac{3 g_{\pi N N}^{2}}{16 \pi^{3}} \int \frac{d^{2} \mathbf{k}_{\perp}}{y^{2}(1-y)} \frac{\mathcal{F}\left(s_{N \pi, i}\right) \mathcal{F}\left(s_{N \pi, f}\right)}{\left(s_{N \pi, i}-M^{2}\right)\left(s_{N \pi, f}-M^{2}\right)} \\
& \times\left(-2 M^{2}\right)(1-y)^{2},
\end{aligned}
$$

where the squared center of mass energies are:

$$
s_{N \pi, i(f)}=s_{\pi N}+\frac{\mathbf{q}_{\perp}}{y} \cdot\left((1-y) \frac{\mathbf{q}_{\perp}}{4} \pm \mathbf{k}_{\perp}\right)
$$




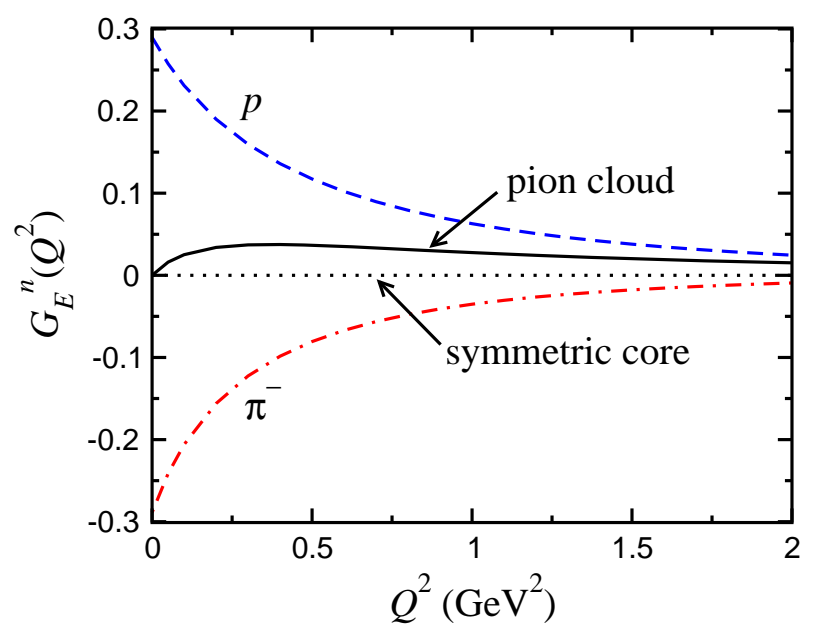

FIG. 2: Neutron electric form factor in the pion cloud model [26]. The direct $\pi^{-}$coupling contribution is represented by the dot-dashed curve, the coupling to the recoil proton by the dashed curve, and the total by the solid. The symmetric core contribution is indicated by the dotted line.

with $s_{\pi N}$ defined in Eq. (7).

The contribution from coupling directly to the pion is:

$$
\begin{aligned}
f_{1}^{(\pi)}\left(y, Q^{2}\right)= & \frac{3 g_{\pi N N}^{2}}{16 \pi^{3}} \int \frac{d^{2} \mathbf{k}_{\perp}}{y^{2}(1-y)} \frac{\mathcal{F}\left(s_{\pi N, i}\right) \mathcal{F}\left(s_{\pi N, f}\right)}{\left(s_{\pi N, i}-M^{2}\right)\left(s_{\pi N, f}-M^{2}\right)} \\
& \times\left(k_{\perp}^{2}+M^{2}(1-y)^{2}-y^{2} \frac{q_{\perp}^{2}}{4}\right), \\
f_{2}^{(\pi)}\left(y, Q^{2}\right)= & \frac{3 g_{\pi N N}^{2}}{16 \pi^{3}} \int \frac{d^{2} \mathbf{k}_{\perp}}{y^{2}(1-y)} \frac{\mathcal{F}\left(s_{\pi N, i}\right) \mathcal{F}\left(s_{\pi N, f}\right)}{\left(s_{\pi N, i}-M^{2}\right)\left(s_{\pi N, f}-M^{2}\right)} \\
& \times\left(2 M^{2} y(1-y)\right),
\end{aligned}
$$

where the $\pi N$ squared center of mass energies are:

$$
s_{\pi N, i(f)}=s_{\pi N}+\frac{\mathbf{q}_{\perp}}{1-y} \cdot\left(y \frac{\mathbf{q}_{\perp}}{4} \pm \mathbf{k}_{\perp}\right) .
$$

The $N \rightarrow \pi N$ splitting functions are related to the distribution functions in Eqs. (10) and (13) by:

$$
\begin{aligned}
& f_{1}^{(\pi)}\left(y, Q^{2}=0\right)=f_{\pi N}(y) \\
& f_{1}^{(N)}\left(y, Q^{2}=0\right)=f_{N \pi}(y)=f_{\pi N}(1-y) .
\end{aligned}
$$

Using the same pion cloud parameters as in the calculation of the $\bar{d} / \bar{u}$ asymmetry in Fig. 1, the relative contributions to $G_{E}^{n}$ from the $\pi^{-}$and recoil proton are shown in Fig. 2. 
Both are large in magnitude but opposite in sign, so that the combined effects cancel to give a small positive $G_{E}^{n}$, consistent with data which show a small and positive $G_{E}^{n}$ in the range $0<Q^{2} \lesssim 1.5 \mathrm{GeV}^{2}$. Note, however, that the Pauli blocking effect plays no role in form factors, since any suppression of $\bar{u}$ relative to $\bar{d}$ here would be accompanied by an equal and opposite suppression of $u_{\text {sea }}$ relative to $d_{\text {sea }}$, and form factors always contain charge conjugation odd (valence) combinations of flavors.

\section{Proton's electric to magnetic form factor ratio}

As well as the neutron's electric form factor, the pion cloud has also been argued to play an important role in the description of the ratio of the proton's electric to magnetic form factors $G_{E}^{p} / G_{M}^{p}$. In particular, models of the pion cloud [28, 29] provide a reasonable description of the fall-off in the ratio at large $Q^{2}$ observed in recent experiments at Jefferson Lab [30], at least up to $Q^{2} \sim 2-3 \mathrm{GeV}^{2}$. Unfortunately, the details of the valence quark core affect the quantitative predictions for the form factors, and at larger $Q^{2}$, where the observed effect is more pronounced, simple valence quark models are no longer reliable.

A more model-independent approach to study the effects of the pion cloud on the nucleon's electromagnetic form factors, at low or high $Q^{2}$, is via lattice QCD. Currently lattice studies are restricted to unphysically large values of $m_{\pi}$, and do not extend beyond $Q^{2} \sim 2 \mathrm{GeV}^{2}$. A novel approach to extract information from currently available lattice data was proposed by Matevosyan et al. [31], using the light-front cloudy bag model [29] to study the pion (or equivalently quark) mass dependence of the nucleon form factors. The lattice data were found to be well reproduced by allowing the parameters of the model be analytic functions of quark mass. These functions were also used to define extrapolations to the physical pion mass and thereby study the variation of the zero crossing of $G_{E}^{p} / G_{M}^{p}$ with the quark mass.

As a final remark, note that the prominent feature of the rapid fall-off with $Q^{2}$ of the $G_{E}^{p} / G_{M}^{p}$ was not evident until the recent experiments using the polarization transfer technique to measure the form factor ratio [30]. Previous data on $G_{E}^{p} / G_{M}^{p}$ were extracted from elastic cross section measurements using the Rosenbluth or longitudinal-transverse (LT) separation technique, which were consistent with a constant behavior with $Q^{2}$ [32]. The res-

olution of the discrepancy between the two sets of measurements came with the realization 

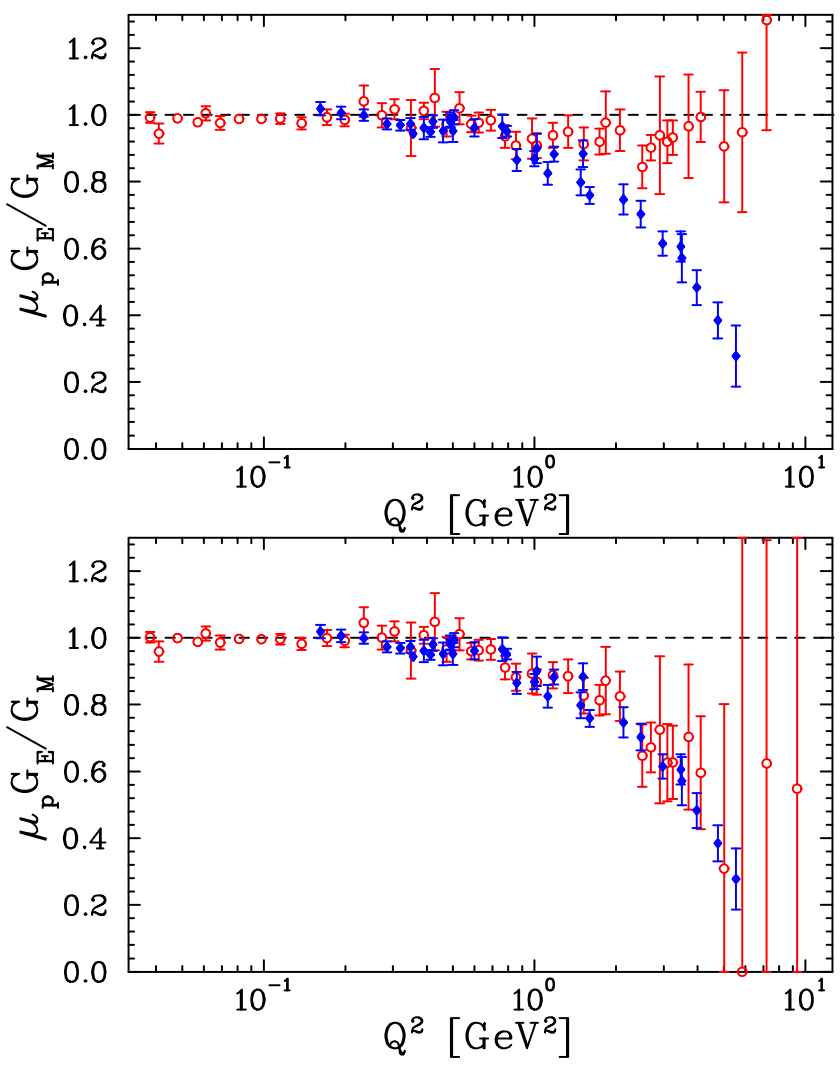

FIG. 3: Ratio $\mu_{p} G_{E}^{p} / G_{M}^{p}$ extracted from polarization transfer (filled diamonds) and LT measurements (open circles), where $\mu_{p}$ is the proton's magnetic moment. The top (bottom) figure shows LT separations without (with) two-photon corrections applied to the cross sections [34].

that corrections from two-photon exchange had a significant impact on the angular dependence of the Rosenbluth cross sections, rendering the LT separation method impractical at large $Q^{2}$. Explicit calculations [33] demonstrated that the sign and magnitude of these corrections was sufficient to explain the bulk of the discrepancy [34], as illustrated in Fig. 3. As an interesting aside, I first met my eventual collaborator on the two-photon exchange work, Prof. J. A. Tjon, at the University of Maryland, where he was a frequent visitor and collaborator of Prof. Banerjee.

\section{Conclusion}

Over the past decade or so a number of experiments and refined data analyses have forced a re-evaluation of our view of the nucleon in terms of three valence quarks immersed in a 
sea of perturbatively generated $q \bar{q}$ pairs and gluons. A new picture has emerged where the nonperturbative structure of the nucleon, in the form of its pion cloud, plays an important role in both the low energy and high energy realms.

In particular, understanding the $\bar{d}-\bar{u}$ flavor asymmetry in the proton sea observed in DIS and Drell-Yan experiments is impossible without the presence of a pion cloud around the nucleon. I have reviewed how insight from the leading non-analytic structure of chiral loops leads to model-independent predictions for the existence of such an asymmetry in QCD. Furthermore, specific meson cloud models of the nucleon are able to reproduce the main features of the data over most of the range of kinematics. An exception is the region of large $x(x \sim 0.3)$, where the apparent downturn in the $\bar{d} / \bar{u}$ data is difficult to accommodate solely in terms of a pion cloud, although the experimental uncertainties in this region are sizable. A new dedicated Drell-Yan experiment at Fermilab [23] may be able to unambiguously determine the sign and magnitude of the $\bar{d}-\bar{u}$ up to $x \sim 0.45$, as could experiments at Jefferson Lab following the $12 \mathrm{GeV}$ Upgrade.

At lower $Q^{2}$ the same pion cloud framework naturally predicts a nonzero charge distribution in the neutron arising from the virtual process $n \rightarrow p+\pi^{+} \rightarrow n$. I showed how in a simple model the large contributions from scattering from the proton core and the $\pi^{-}$ cloud cancel, leaving a small net positive $G_{E}^{n}$ form factor peaking at around $Q^{2} \sim 0.4 \mathrm{GeV}^{2}$. Furthermore, the possible role of the pion cloud has been discussed in the proton's electric to magnetic form factor ratio, which was recently found to have a surprisingly strong $Q^{2}$ dependence between $Q^{2} \sim 1$ and $6 \mathrm{GeV}^{2}$.

Extending these ideas to the strangeness sector is also possible. As originally noticed by Signal and Thomas [35], virtual kaon loops are one possible source of nonperturbative strangeness in the nucleon [36]. This has also been placed on a firm footing by observing that the moments of the difference between the $s$ and $\bar{s}$ distributions in the nucleon are nonanalytic functions of $m_{q}+m_{s}$, with $m_{s}$ the strange quark mass, leading to model-independent predictions for the asymmetry between $s$ and $\bar{s}$ in the nucleon sea. Future higher-quality neutrino DIS data should help establish the sign and magnitude of this asymmetry. Finally, strange chiral loops can also lead to nonzero values for strange electric and magnetic form factors [36]. The magnitude of the kaon cloud contributions, although rather small, is consistent with that of the available data [37]. 


\section{Acknowledgments}

This work was supported by the DOE contract No. DE-AC05-06OR23177, under which Jefferson Science Associates, LLC operates Jefferson Lab.

[1] M. K. Banerjee, Prog. Part. Nucl. Phys. 31, 77 (1993).

[2] A. W. Thomas and W. Melnitchouk, in New Frontiers in Nuclear Physics (World Scientific, 1993).

[3] A. W. Thomas, W. Melnitchouk and F. M. Steffens, Phys. Rev. Lett. 85, 2892 (2000).

[4] D. Arndt and M. J. Savage, Nucl. Phys. A 697, 429 (2002).

[5] J. W. Chen and X. d. Ji, Phys. Lett. B 523, 107 (2001).

[6] W. Melnitchouk, in Hadronic Structure, 14th Annual HUGS at CEBAF, ed. J. Goity (World Scientific, 2001), arXiv:hep-ph/0006170.

[7] M. A. B. Bég and A. Zepeda, Phys. Rev. D 6, 2912 (1972); J. Gasser, M. E. Sainio and A. Svarc, Nucl. Phys. B307, 779 (1988); D. B. Leinweber and T. D. Cohen, Phys. Rev. D 47, 2147 (1993).

[8] Note that from the Gell-Mann-Oakes-Renner relation $m_{\pi}^{2} \sim m_{q} \equiv\left(m_{u}+m_{d}\right) / 2$.

[9] J. D. Sullivan, Phys. Rev. D 5, 1732 (1972).

[10] A. W. Thomas, Phys. Lett. B 126, 97 (1983).

[11] P. Amaudraz et al., Phys. Rev. Lett. 66, 2712 (1991).

[12] A. Baldit et al., Phys. Lett. B 332, 244 (1994).

[13] E. A. Hawker et al., Phys. Rev. Lett. 80, 3715 (1998).

[14] J.-C. Peng and G. T. Garvey, in Trends in Particle and Nuclear Physics, (Plenum Press, New York, 1999), arXiv:hep-ph/9912370.

[15] For reviews see: J. Speth and A.W. Thomas, Adv. Nucl. Phys. 24, 83 (1998); S. Kumano, Phys. Rep. 303, 183 (1998).

[16] S. Weinberg, Physica (Amsterdam) 96 A, 327 (1979); J. Gasser and H. Leutwyler, Ann. Phys. 158, 142 (1984).

[17] W. Melnitchouk, J. Speth and A.W. Thomas, Phys. Rev. D 59, 014033 (1999).

[18] S. Théberge, G. A. Miller and A. W. Thomas, Can. J. Phys. 60, 59 (1982). 
[19] S. Weinberg, Phys. Rev. 150, 1313 (1966); S. D. Drell, D. J. Levy, and T. M. Yan, Phys. Rev. D 1, 1035 (1970).

[20] W. Melnitchouk and A. W. Thomas, Phys. Rev. D 47, 3794 (1993).

[21] A. W. Schreiber, A. I. Signal and A. W. Thomas, Phys. Rev. D 44, 2653 (1991).

[22] R. D. Field and R. P. Feynman, Phys. Rev. D 15, 2590 (1977).

[23] Fermilab experiment E906, P. Reimer et al. spokespersons.

[24] J. Levelt, P. J. Mulders and A. W. Schreiber, Phys. Lett. B 263, 498 (1991).

[25] K. Ackerstaff et al., Phys. Rev. Lett. 81, 5519 (1998).

[26] W. Melnitchouk, in Proceedings of the Workshop on Exclusive ES Semi-Exclusive Processes at High Momentum Transfer (Jefferson Lab, 1999), arXiv:hep-ph/9909463.

[27] N. Isgur, G. Karl and D. W. L. Sprung, Phys. Rev. D 23, 163 (1981).

[28] D. H. Lu, A. W. Thomas and A. G. Williams, Phys. Rev. C 57, 2628 (1998).

[29] G. A. Miller, Phys. Rev. C 66, 032201 (2002).

[30] M. K. Jones et al., Phys. Rev. Lett. 84, 1398 (2000); O. Gayou et al., Phys. Rev. Lett. 88, 092301 (2002); V. Punjabi et al., Phys. Rev. C 71, 055202 (2005) [Erratum-ibid. C 71, 069902 (2005)].

[31] H. H. Matevosyan, G. A. Miller and A. W. Thomas, Phys. Rev. C 71, 055204 (2005).

[32] R. C. Walker et al., Phys. Rev. D 49, 5671 (1994).

[33] P. G. Blunden, W. Melnitchouk and J. A. Tjon, Phys. Rev. Lett. 91, 142304 (2003); Phys. Rev. C 72, 034612 (2005).

[34] J. Arrington, W. Melnitchouk and J. A. Tjon, Phys. Rev. C 76, 035205 (2007).

[35] A. I. Signal and A. W. Thomas, Phys. Lett. B 191, 206 (1987).

[36] W. Melnitchouk and M. Malheiro, Phys. Rev. C 55431 (1997); M. Malheiro and W. Melnitchouk, Phys. Rev. C 562373 (1997); W. Melnitchouk and M. Malheiro, Phys. Lett. B 451, 224 (1999).

[37] R. D. Young, J. Roche, R. D. Carlini and A. W. Thomas, Phys. Rev. Lett. 97, 102002 (2006) 